kamal, \&c., it was difficult to believe that it did not exist or that such a device was not used, especially as the water level could be said to act as an artificial horizon.

Admiral Rodman wrote that the specimen he had tried resided at the Bishop Museum of Hawaii. However, although there is such a specimen there, it is not an instrument of navigation and neither is it described as such. It is a container for valuable possessions, or in other words, a watertight suitcase to use on long voyages. It has, indeed, a series of holes around the rim and they are in threes, but their distance from the edge is such that sighting through even the lowest of the three holes would only give an angle of $\mathrm{I}^{\circ}$ or thereabouts. In no case do the holes give an angle anywhere near $20^{\circ}$. Moreover, the height of the calabash is some 33 inches, with perhaps a 2 inch diameter. Filled with water to the hole level would give a weight of water near to $100 \mathrm{lb}$. It might seem then that to hold this weight at chest level, in a bobbing canoe, would present a gargantuan task.

There are, in fact, other similar calabashes (at the Bernice Bishop Museum) which indicate that the use of the holes was probably for threading the lashings through when tying the top on.

It would appear therefore that the 'sacred calabash sextant' is unfortunately a myth and the Polynesians did not, after all, navigate by coconut shells. Fortunately, the sticks of the Marshall Islanders are still with us.

1 E. de Bisschop, Tahiti Nul.

REFEREN CES

2 Admiral Hugh Rodman, U.S.N., Yarns of a Kentucky Admiral.

Correspondence research: Bernice Bishop Museum; University of Hawaii Museum; National Maritime Museum, Honolulu.

\title{
Accuracy Contours for Horizontal Angle Position Lines
}

\author{
E. M. Goodwin and J. F. Kemp \\ (City of London Polytechnic)
}

THIs paper describes a method of constructing accuracy contours for a position line obtained by observing the horizontal angle between two stations. An example is given using an estimate made, from experimental evidence, of the observational errors that are likely to occur when such angles are measured by sextant. A recent paper by Cotter ${ }^{1}$ described the historical development of this method of obtaining a position line.

In principle, the observed horizontal angle $(\theta)$ defines a position line which is the locus of a point that moves so that the angle at the point subtended by the baseline between the two stations is constant. Such a position line is the arc of a circle which passes through the two stations. Its centre may be found since it lies on the perpendicular bisector of the baseline, and the angle at the centre subtended by the baseline is twice the observed angle (Fig. I). 


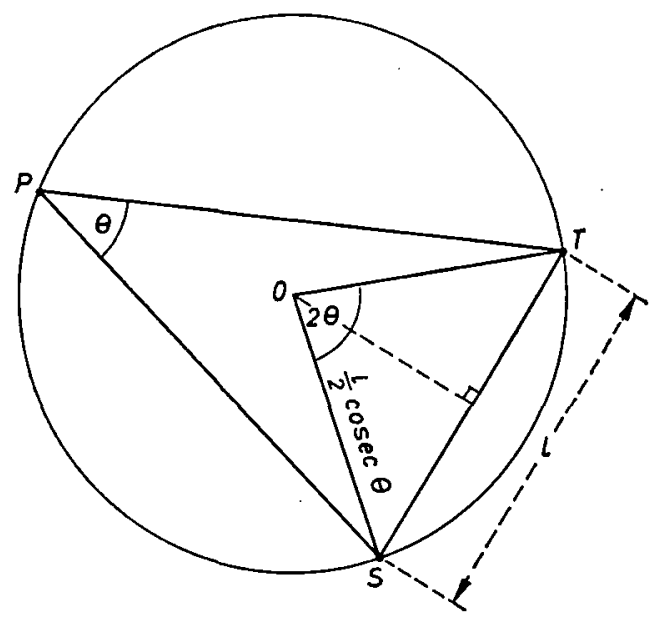

FIG. I

As a first step, an expression is derived for the displacement of an arc of position line due to a given error in an observed angle.

In Fig. 2, $\mathrm{S}$ and $\mathrm{T}$ are two stations with baseline of length $1 . \mathrm{P}$ is a typical point on a position circle defined by an angle $\theta$ subtended between $S$ and $T, O$ being the centre of the position circle. A second position circle defined by an

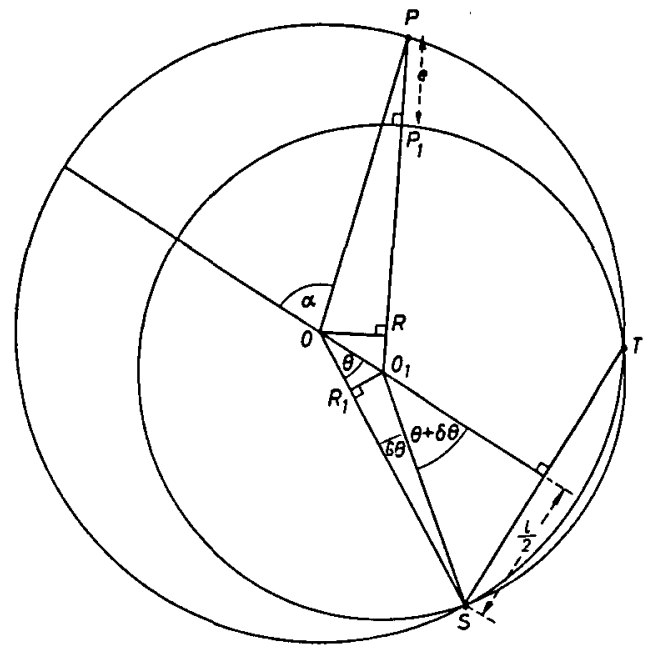

FIG. 2

angle $(\theta+\delta \theta)$ subtended between $S$ and $T$ is drawn with centre at $O_{1}$. From point $\mathrm{P}$, the displacement ( $e$ ) of a short arc of position line due to an increment $\delta \theta$ in the observed angle is $\mathrm{PP}_{1} . \mathrm{R}$ is the foot of a perpendicular drawn from $\mathrm{O}$ on to $\mathrm{PO}_{1}, \mathrm{R}_{1}$ is the foot of a perpendicular drawn from $\mathrm{O}_{1}$ on to OS. The angle between $O P$ and $\mathrm{O}_{1} \mathrm{O}$ produced is designated $\alpha$. 
No. 4

$$
\begin{aligned}
e=P_{1} & =P R-P_{1} R \\
& =P R+\mathrm{RO}_{1}-\left(\mathrm{P}_{1} \mathrm{R}+\mathrm{RO}_{1}\right) \\
& =\mathrm{PO} \cos \angle \mathrm{OPR}+\mathrm{RO}_{1}-\mathrm{P}_{1} \mathrm{O}_{1} \\
& \simeq \mathrm{PO}+\mathrm{RO}_{1}-\mathrm{P}_{1} \mathrm{O}_{1} \quad \text { [since } \angle \mathrm{OPR} \text { is small] } \\
& =\mathrm{OS}-\mathrm{O}_{1} \mathrm{~S}+\mathrm{RO}_{1} \quad \quad \text { [since } \delta \theta \text { is small] } \\
& =\mathrm{OR}_{1}+\mathrm{R}_{1} \mathrm{~S}-\mathrm{O}_{1} \mathrm{~S}+\mathrm{RO}_{1} \\
& =\mathrm{OR}_{1}+\mathrm{O}_{1} \mathrm{~S} \cos \delta \theta-\mathrm{O}_{1} \mathrm{~S}+\mathrm{RO}_{1} \\
& \simeq \mathrm{OR}_{1}+\mathrm{RO}_{1} \quad \cos \angle \mathrm{RO}_{1} \mathrm{O} \\
& =\frac{1}{2} \operatorname{cosec}(\theta+\delta \theta) \delta \theta \cot \theta+\frac{1}{2} \operatorname{cosec}(\theta+\delta \theta) \angle \delta \theta \operatorname{cosec} \theta \\
& =\frac{1}{2} \operatorname{cosec}(\theta+\delta \theta) \delta \theta\left[\cot \theta+\operatorname{cosec} \theta \cos \mathrm{R} \angle \mathrm{O}_{1} \mathrm{O}\right]
\end{aligned}
$$

In practical work, $\delta \theta$ is always very small compared with $\theta$ so that $\operatorname{cosec}(\theta+\delta \theta)$ $\bumpeq \operatorname{cosec} \theta$. For values of $\theta>10^{\circ}$ and $\delta \theta<\mathrm{r} 2^{\prime}$, this approximation is less than 2 per cent in error. Also, when $\delta \theta$ is very small, $\angle \mathrm{RO}_{1} \mathrm{O}=\alpha-\angle \mathrm{OPR} \bumpeq \alpha$. The formula for $e$ thus becomes:

$$
e=\frac{1}{2} \operatorname{cosec}^{2} \theta \delta \theta[\cos \theta+\cos \alpha]
$$

It can be shown similarly that the expression holds when $\delta \theta$ is a decrement.

An error contour is the locus of a point which moves so that $e$ is a constant for given values of 1 and $\delta \theta$. Thus for any particular contour:

$$
\frac{2 e}{1 \delta \theta}=A \quad \text { [where } A \text { is a constant] }
$$

Equation (I) becomes :

$$
A=\operatorname{cosec}^{2} \theta[\cos \theta+\cos \alpha]
$$

Let $\cos \alpha=C$, then

$$
\begin{gathered}
A \sin ^{2} \theta=\cos \theta+C \\
\therefore A\left(1-\cos ^{2} \theta\right)=\cos \theta+C \\
A \cos ^{2} \theta+\cos \theta+C-A=0
\end{gathered}
$$

Equation (2) may be solved to find values of $\theta$ corresponding to a number of chosen values of $\alpha$. The series of points defined by the paired values of $\theta$ and $\alpha$ may then be plotted as the intersections of arcs and radials respectively from centres, of which $O$ in Fig. 2 is typical. The curve joining the points forms the required error contour.

In a practical example the length of the baseline between the two stations $(l)$ is known. The accuracy with which horizontal angles can be measured under the field conditions must also be known in order to define the observational error $\delta \theta$. This may be estimated from experience or from specially designed experiments.

For this paper, observational accuracy was estimated from an experiment in which 32 experienced navigating officers each measured the horizontal angle 


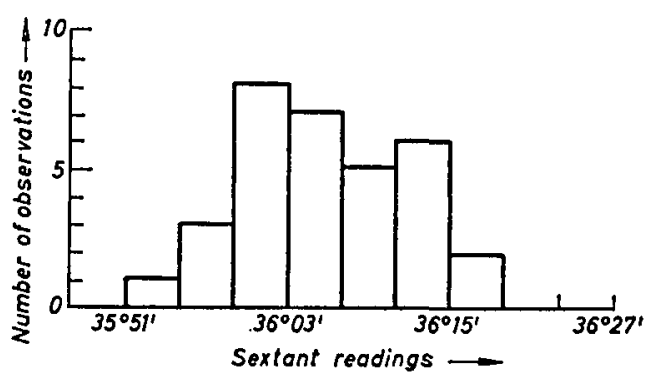

FIG. 3

subtended between two well defined stations. The same sextant was used for all the observations to eliminate instrumental error and readings were taken to the nearest 10 sec. of arc. The observations had a mean value of $36^{\circ} \circ 5^{\prime} 3 \mathrm{I}^{\prime \prime}$ and a standard deviation of $6^{\prime} \circ 5^{\prime \prime} 5$. Fig. 3 is a histogram showing the distribution of the readings.

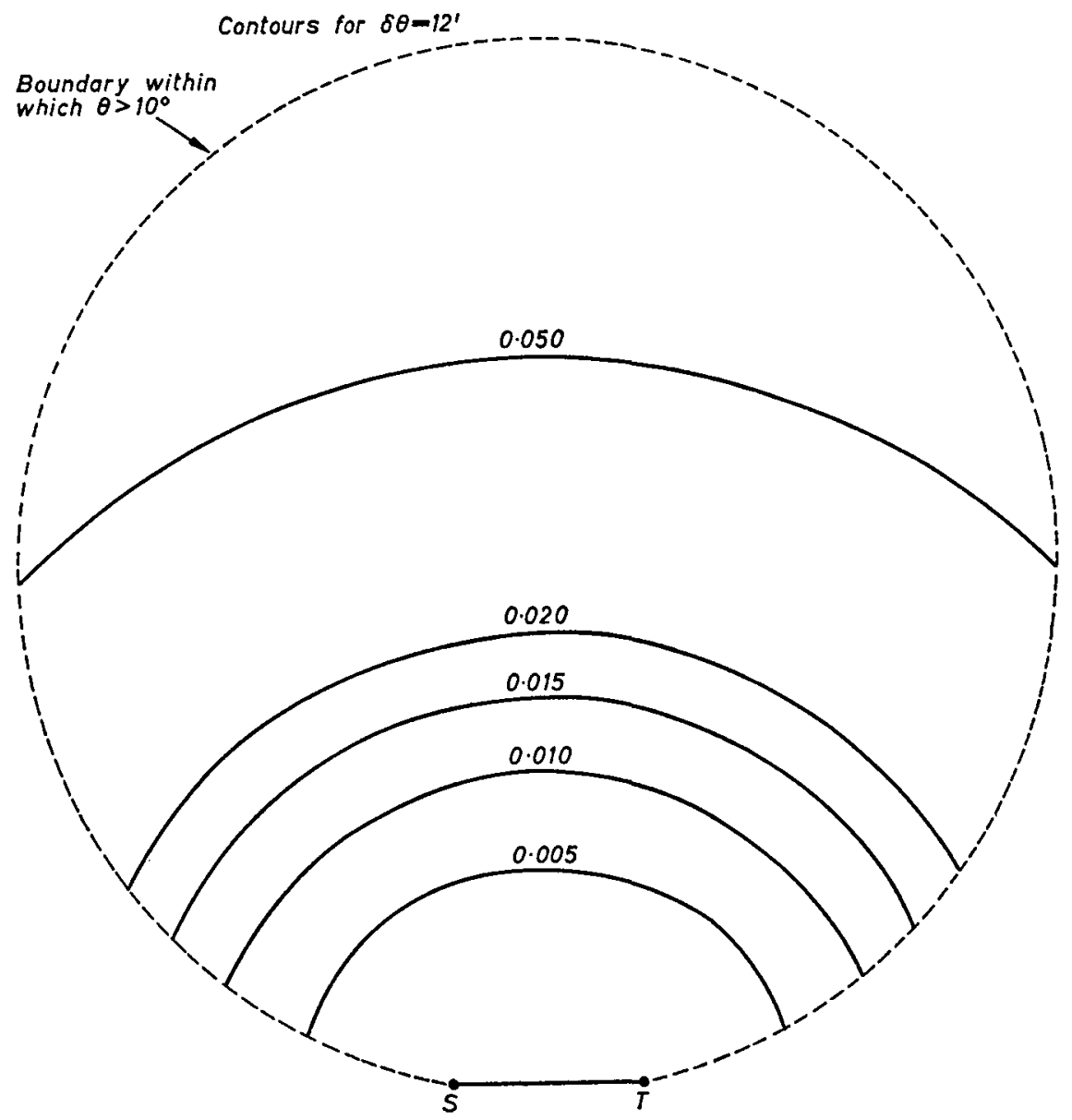

FIG. 4 
If it is assumed that the distribution of error follows a normal distribution in practice then 95 per cent of readings will be within approximately two standard deviations of the true value. In this example therefore 95 per cent of errors are less than $12^{\prime}$. Taking $\delta \theta$ to be $12^{\prime}$ it is thus possible to construct error contours for specified values of the displacement (e). These are known as the 95 per cent error contours. In practical work they are often the most useful, since for points along the contour only one out of twenty observations may be expected to give arcs of position lines which are displaced from the true position line by more than the nominal displacement error $(e)$ of the contour.

It is worth noting that the value of $\mathrm{I}^{\prime}$ for $\delta \theta$ applies to the experimental conditions which are considered to have been rather better than the general case at sea. The observers had a steady platform and unlimited time for their observations. The stations observed were the well defined vertical side of a rectangular tower and a flag pole. Some errors were due to the sextant being held in other than a horizontal plane.

In Fig. 4, taking $\delta \theta$ to be $12^{\prime}$, representative contours are drawn for an area within which the angle $(\theta)$ subtended by the two stations ( $S$ and $T$ ) is greater than $10^{\circ}$. The values of position line displacement $(e)$ for which the contours are drawn are given as decimals of the baseline length so that the diagram may be used for any required length of baseline. Thus, for a baseline of 1000 metres, the $0.005,0.010$ and $0.015,0.020$ and 0.050 contours would represent the 5 metre, 10 metre, 15 metre, 20 metre and 50 metre contours respectively.

The contours discussed in this paper are useful for defining the accuracy coverage of horizontal angle position lines as affected by random observational errors. The effect of systematic errors in observed angles should be dealt with by other methods. In particular, the Admiralty Manual of Hydrographic Surveying gives a very full treatment of errors due to the stations observed being on different levels.

\section{REFEREN CE}

1 Cotter, C. H. (1972). A brief history of the method of fixing by horizontal angles. This Journal, 25, 528

\section{Martelli's Tables}

\section{Charles H. Cotter}

Captain S. T. L. Lecky, in his famous Wrinkles, 1 wrote with derision in his reference to so-called 'short-methods'. He warned his readers to 'beware' of these :

They generally only look short [wrote Lecky] because good care is taken to apply the various corrections beforehand, and the unsuspecting reader is deceived of this device.

As a case in point Lecky considered the 'small but expensive pamphlet by Mr. Martelli', to support his derogatory remarks :

When his so-called 'short-method' is overhauled and compared with Raper, we get the following startling result:-Martelli, 56 figures and five logarithms, 